\title{
Region similarity based edge detection for motion estimation in H.264/AVC
}

\author{
Anand Paul $^{1 \mathrm{aa})}$, K. Bharanitharan ${ }^{2 \mathrm{~b})}$, and Jhing-Fa Wang ${ }^{3 \mathrm{c})}$ \\ ${ }^{1}$ Department of Electronics Engineering, Hanyang University, Seoul, South Korea \\ ${ }^{2}$ Department of Electrical Engineering, Korea University, Seoul, South Korea \\ ${ }^{3}$ Department of Electrical Engineering, National Cheng Kung University, \\ \#1, Dasyue Road, Tainan - 701, Taiwan \\ a)anand@hanyang.ac.kr \\ b)dharan@korea.ac.kr \\ c)wangif@csie.ncku.edu.tw
}

Abstract: This paper introduces a block-based motion estimation algorithm based on projection with adaptive window size selection. By using one-dimensional (1-D) projection the original two-dimensional (2-D) block-matching problem is translated to a simpler 1D matching, which eliminates majority of potential pixel participation. In this paper, we propose an efficient fast motion estimation method by using region similarity detection (RSD) algorithm to reduce the encoding time. The proposed RSD algorithm effectively estimates the texture direction of the block to find the direction and then horizontal, vertical or diagonal projection is employed for SAD calculation. Experimental results show that the proposed algorithm achieves more than a $60 \%$ time reduction with negligible PSNR loss and bitrate increments.

Keywords: motion estimation, H.264/AVC, edge detection, projection

Classification: Science and engineering for electronics

\section{References}

[1] T. Wiegand, G. J. Sullivan, G. Bjontegaard, and A. Luthra, "Overview of the H.264/AVC video coding standard," IEEE Trans. Circuits Syst. Video Technol., vol. 13, no. 7, pp. 560-576, July 2003.

[2] C. Tu, T. Tran, and P. Topiwala, "A hybrid feature/image block motion estimation approach," ITU-T/VCEG M.26.doc, Austin Meeting, April 2001.

[3] G. Bjontegarrd, "Calculation of Average PSNR Difference between RDcurve," ITU-TSC16/Q6, 13th VCEG Meeting, Austin, Texas, April 2001.

[4] C. Zhu, X. Lin, and L.-P. Chau, "Hexagonal-based search pattern for fast block motion estimation," IEEE Trans. Circuits Syst. Video Technol., vol. 12, no. 15, pp. 349-355, May 2002.

[5] Z. Chen, Y. Song, T. Ikenaga, and S. Goto, "Adaptive Search Range Algorithm for Variable Block Size Motion Estimation in H.264/AVC," 


\section{Introduction}

For the past twenty years several video coding standards such as MPEG-1, MPEG-2/4, H.263, H.264 [1], have been playing an significant role in digital media revolution. The recent advancement in H.264 significantly increases coding efficiency and processor computation power with inclusion of variable block motion estimation and many other coding features. Computation complexity in advanced video encoders is mainly contributed by motion estimation, which can effectively remove the temporal redundancy in video data. The computational complexity of block-based motion estimation is the main consequence of the expensive two-dimensional (2-D) block matching process. In this paper, a projection-based adaptive fast motion estimation algorithm is proposed. Generally, the blocks cannot match well if its corresponding one-dimensional (1-D) projection does not match well. Thus, the 2-D block matching problem can be transformed to a simpler 1-D matching problem, which eliminates majority of all potential pixels participation [2]. Before projection we are to determine the motion flow in horizontal, vertical or diagonal direction. In this paper, a bi-region similarity detection (BSD) method, which only needs to compute the mean and variance of two split regions to find the direction of flow, is proposed. The BSD method makes the detection algorithm simple enough to greatly reduce the computation time with less than $0.1 \mathrm{~dB}$ PSNR degradation and a negligible bit rate decrement.

The remaining of this paper is organized as follows. Section 2 introduces the projection-based motion estimation. In Section 3, Region similarity algorithm is elaborated. Adaptive search range selection method is discussed in section 4. Performance evaluation of the proposed scheme is presented in Section 5. Finally, conclusion is addressed in Section 6.

\section{Projection based Motion Estimation}

Horizontal, vertical or diagonal projections are performed only after motion direction is detected. For simplicity, only vertical projection is discussed in this section.

If the frame size is $W \times H$, the block size is $B_{h} \times B_{v}$, and the search range size is $R_{h} \times R_{v}$, then to predict one block in integer pixel resolution, there are $R_{h} \times R_{v}$ candidate blocks to be searched in the reference frame. For most standards, the common block size, which is also known as the macroblock, is $16 \times 16$.

Let $b_{i, j}^{x, y}$ be the pixel value at the $j^{\text {th }}$ row and $i^{\text {th }}$ column of the block $\boldsymbol{B}^{x, y}$ located at the $(x, y)$ coordination for $0 \leq i<B_{h}$, and $0 \leq j<B_{v}$. The vertical (column) projection of $\boldsymbol{B}^{x, y}$ is defined as $\boldsymbol{P}_{B}^{x, y}$,, which is a 1-D row vector where the $i^{\text {th }}$ element is the sum of the $i^{\text {th }}$ column of $\boldsymbol{B}^{x, y}$. Thus, the $j^{\text {th }}$ element of $\boldsymbol{P}_{B}^{x, y}$ can be expressed by: 


$$
p_{B, i}^{x, y}=\sum_{j=0}^{B_{v}-1} b_{i, j}^{x, y}, \quad 0 \leq i<B_{h}, \text { and } 0 \leq j<B_{y} .
$$

By the vertical projection, a $B_{h} \times B_{v}$ 2-D block is reduced to a $B_{h}$ component vector, and only DC information of each column is preserved [10] as shown in Fig. 1.

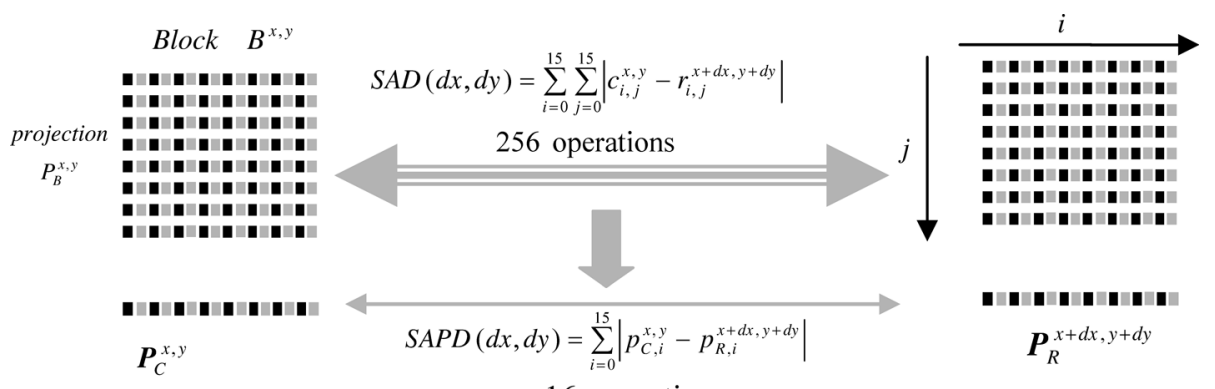

16 operations

Fig. 1. Comparison of 2-D matching cost (SAD) and 1-D matching cost (SAPD)

For each block with proper buffering, only 2 operations per pixel are required on average to compute the projection of a block in this updating manner. The cost for projection is thus only $\mathrm{O}(2 W \times H)$ operations.

For a block $C^{x, y}$ in the current frame, block based motion estimation searches all displaced blocks $\boldsymbol{R}^{x+d x, y+d y}$ in the search window in the reference frame for the best-matched block. The commonly used matching error metric called sum of absolute difference (SAD) is given by:

$$
S A D(d x, d y)=\sum_{i=0}^{B_{h}-1} \sum_{j=0}^{B_{v}-1}\left|c_{i, j}^{x, y}-r_{i, j}^{x, y}\right|
$$

However, the matching error after 1-D projections becomes the sum of absolute projection difference (SAPD) between blocks $\boldsymbol{C}^{x, y}$ and $\boldsymbol{R}^{x+d x, y+d y}$ is

$$
S A P D(d x, d y)=\sum_{i=0}^{B_{h}-1}\left|p_{C, i}^{x, y}-p_{R, i}^{x+d x, y+d y}\right| .
$$

The SAD computation stated in Eq. (2) acquires more operations comparing to the SAPD depicted in Eq. (3). For computation of the matching errors, we can see that 256 operations by $2-\mathrm{D}$ matching are reduced to 16 operations by $1-\mathrm{D}[2]$.

\section{Region Similarity Detection}

\subsection{Region similarity detection for $4 \times 4$ block}

Figure 2 brings out the different region. As pattern 1 detect 90 degree shift, pattern2 detect zero degree shift edge and so on. The Region similarity detection algorithm is expressed in following steps. 


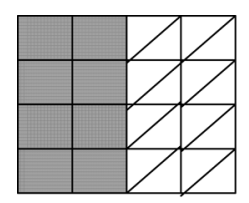

(Pattern1)

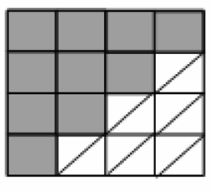

45

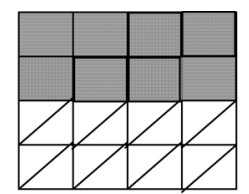

(Pattern2)

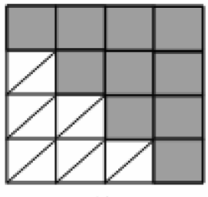

135
Region 1

Region 2

\section{(Patter3) (Pattern4)}

Fig. 2. Four different patter for $4 \mathrm{X} 4$ Block

Step 1: Divide the $4 \times 4$ block into Regions 1 and 2 for each mode as shown in Fig. 2.

Step 2: Compute the means for Regions 1 and 2 using;

$$
\mu_{k}=\frac{1}{\left|R_{k}\right|} \sum_{(i, j) \in R_{k}} x(i, j) \text { for } k=1 \text { and } 2
$$

where $x(i, j)$ denotes the pixel value and $\left|R_{k}\right|$ represents the total number of pixels in the $k^{\text {th }}$ region.

Step 3: Compute the variances of Regions 1 and 2 using;

$$
\sigma_{k}^{2}=\frac{1}{\left|R_{k}\right|} \sum_{(i, j) \in R_{k}}\left(x(i, j)-\mu_{k}\right)^{2} \text { for } k=1 \text { and } 2
$$

Step 4: Add the variances of Regions 1 and 2 together to obtain the total roughness using;

$$
r_{\text {mode }}=\sigma_{1}^{2}+\sigma_{2}^{2}
$$

Step 5: Repeat Step 1 to Step 4 for all 4 designed patterns.

Step 6: Select the pattern with the minimum roughness as the direction (0 degree, or 90 degree or 45 degree (Diagonal) or 135 degree (diagonal)

\subsection{Region similarity detection for $16 \times 16$ block}

Step 1: We need to take average using Eq. (7) after this average then we divide the patterns in to region 1 and 2 as like $4 \times 4$ block then decide the direction.

$$
I_{16 \times 16}=\sum_{k=0}^{3} \sum_{l=0}^{3} f(a+k, b+l) / 16
$$

Where $a$ and $b$ indicate the Starting position luma block.

Step 2: After average, all the above procedures (Step 1 to 6) are the same for $16 \mathrm{X} 16$ block.

After determining the edge direction, vertical, horizontal or diagonal projection is preformed depending up on the edge direction. 


\section{Adaptive Search Range Selection}

In the adaptive search range (ASR) is equated to the arithmetical average of all MVs in previous five reference frames. In window size selection, the average SADs, (Avg_SAD) is obtained from the ROB is used to determine the threshold of adaptive search window size. After statistical analyses, we choose thresholds $\theta_{1}=$ Avg_SAD $_{\text {and }} \theta_{2}=1.2$ Avg_SAD. Without lose of generality, the search window for any given block is chosen from three candidate search regions, i.e., $\pm 32, \pm 16$, and \pm 8 pixels. The proposed algorithm optimally varies the search window region. If $\mathrm{SAD}$ of a macroblock $\left(\mathrm{SAD}_{\mathrm{MB}}\right)$ is less than the first threshold $\left(\theta_{1}\right)$, the $1^{\text {st }}$ window size $( \pm 8)$ is selected for the current frame. If $\mathrm{SAD}_{\mathrm{MB}}$ is greater than the second threshold, $\theta_{2}$, the $2^{\text {nd }}$ window size is selected for the current frame similarly for the $3^{\text {rd }}$ window size.

In summary, the determination could be stated as:

$$
\begin{aligned}
& S A D_{\mathrm{MB}}<\theta_{1}, \\
& \theta_{2}>S A D_{\mathrm{MB}}>\theta_{1}, \\
& S A D_{\mathrm{MB}}>\theta_{2},
\end{aligned}
$$

where $S A D_{\mathrm{MB}}$ the denotes the SAD of current macroblock.

\section{Performance evaluations}

Our simulation results show that the proposed method reduces the encoding time significantly along with bitrate and thus computation complexity drastically reduced and finds the motion vector quickly, which increases encoding speed. When we have smaller motion within the frame larger search range is not chosen which also contributes in reducing computation.

$$
\Delta \mathrm{TIME}=\frac{T_{\text {method }}-T_{\text {ref }}}{T_{\text {ref }}} \times 100 \%
$$

Where $\mathrm{BR}_{\text {method }}$ and $\mathrm{T}_{\text {method }}$ are proposed birtate and time where as $\mathrm{BR}_{\text {ref }}$ and $\mathrm{T}_{\text {ref }}$ are JM reference software bitrate and time. Two delta measurement parameters, $\triangle$ PSNR, $\triangle$ Bitrate (BR) are defined in [3], are used to evaluate encoding performance and are tabulated in Table I with different quantization parameter (28, 32, 36 and 40). Experiment was done for both QCIF and CIF sequences proposed method is compared with hexagonal search and Chen's method.

\section{Conclusion}

In this paper, we have presented region similarity detection algorithm which can detect the edge. Once the edge direction is detected 2D block matching problem is converted to $1 \mathrm{D}$ which reduces the computational complexity involved in motion estimation. Finally an adaptive search range selection algorithm is proposed which adaptively change the search range depending up on the video content. Our experimental results shows that our proposed 
Table I. Comparison for CIF and QCIF sequence with Qp $(28,32,36,40)$

\begin{tabular}{|c|c|c|c|c|c|c|c|c|c|c|}
\hline \multirow{7}{*}{ CIF } & \multirow{2}{*}{ Sequence } & \multicolumn{3}{|c|}{ [4] } & \multicolumn{3}{|c|}{ [5] } & \multicolumn{3}{|c|}{ Proposed } \\
\hline & & $\begin{array}{c}\mathrm{PSNR} \\
{[\mathrm{dB}]} \\
\end{array}$ & $\begin{array}{c}\mathrm{BRR} \\
{[\%]} \\
\end{array}$ & $\begin{array}{l}\Delta \mathrm{T} \\
{[\%]} \\
\end{array}$ & $\begin{array}{c}\mathrm{PSNR} \\
{[\mathrm{dB}]} \\
\end{array}$ & $\begin{array}{l}\Delta \mathrm{BR} \\
{[\%]} \\
\end{array}$ & $\begin{array}{l}\mathrm{T} \\
{[\%]} \\
\end{array}$ & $\begin{array}{c}\mathrm{PSNR} \\
{[\mathrm{dB}]} \\
\end{array}$ & $\begin{array}{l}\Delta \mathrm{BR} \\
{[\%]} \\
\end{array}$ & $\begin{array}{l}\mathrm{T} \\
{[\%]} \\
\end{array}$ \\
\hline & Foreman & -0.200 & 3.220 & -57.80 & -0.275 & 4.121 & -62.00 & -0.167 & 2.323 & -75.45 \\
\hline & Mobile & -0.244 & 3.162 & -59.32 & -0.191 & 2.432 & -59.06 & -0.115 & 1.800 & -74.25 \\
\hline & Tempete & -0.240 & 3.588 & -57.58 & -0.187 & 3.121 & -60.52 & -0.169 & 2.502 & -73.66 \\
\hline & Coastguard & -0.241 & 3.707 & -57.95 & -0.235 & 3.064 & -62.66 & -0.206 & 2.661 & -75.54 \\
\hline & Average & -0.231 & 3.419 & -58.16 & -0.222 & 3.184 & -61.06 & -0.164 & 2.321 & -74.72 \\
\hline \multirow{5}{*}{ QCIF } & Foreman & -0.271 & 4.412 & -65.34 & -0.088 & 1.678 & -69.89 & -0.213 & 4.301 & -78.73 \\
\hline & Mobile & -0.262 & 3.676 & -56.12 & -0.284 & 4.914 & -63.49 & -0.140 & 2.809 & -78.88 \\
\hline & Paris & -0.190 & 3.535 & -64.18 & -0.161 & 3.823 & -57.45 & -0.174 & 3.800 & -75.72 \\
\hline & Silent & -0.102 & 2.378 & -52.11 & -0.202 & 4.330 & -63.80 & -0.166 & 2.932 & -76.91 \\
\hline & Average & -0.206 & 3.584 & -58.39 & -0.183 & 3.931 & -63.35 & -0.183 & 3.436 & -77.43 \\
\hline
\end{tabular}

method out perform the standard JM fast algorithm for motion estimation and other popular existing fast algorithms by over $60 \%$ reduction in computation time with negligible PSNR degradation.

\section{Acknowledgments}

This work was supported in part by the Korea University under Newly Appointed Faculty Research Grant. 